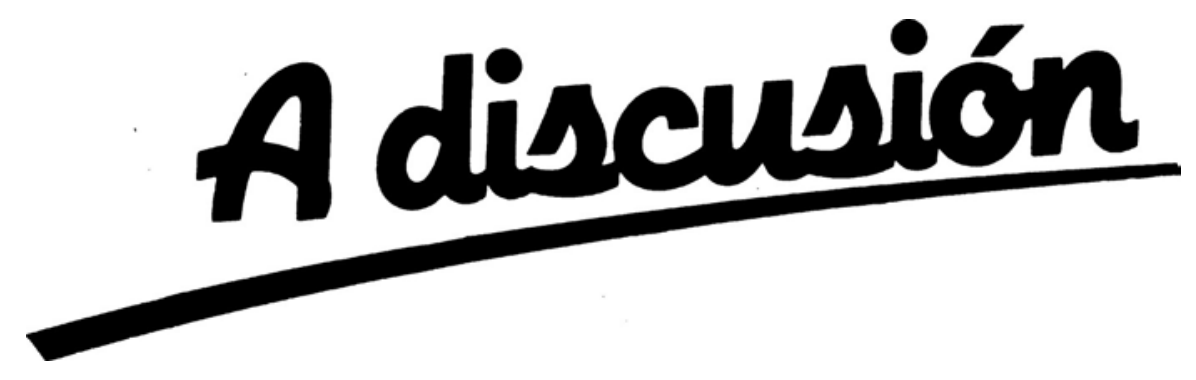

\title{
TWO-STAGE QUANTILE REGRESSION WHEN THE FIRST STAGE IS BASED ON QUANTILE REGRESSION*
}

\section{Tae-Hwan Kim and Christophe Muller**}

\author{
WP-AD 2004-03
}

Corresponding author: T.H. Kim: School of Economics, University of Nottingham, Nottingham NG7 2RD, U.K., Tae-Hwan.Kim@nottingham.ac.uk.

Editor: Instituto Valenciano de Investigaciones Económicas, S.A.

Primera Edición Enero 2004.

Depósito Legal: V-512-2004

IVIE working papers offer in advance the results of economic research under way in order to encourage a discussion process before sending them to scientific journals for their final publication.

\footnotetext{
* This is a revised version of the Notthingham Discussion Paper with the same title. We are grateful to R. Davidson, W. Newey, J. Powell, H. White and O. Linton for useful discussions about a preliminary working paper and to participants in presentations in Nottingham University, CREST-INSEE in Paris, London School of Economics, University of California at San Diego, the 1999 Econometric Study Group Meeting in Bristol, the 2000 Royal Statistical Society Conference in Reading. We also would like to thank two referees for comments and suggestions, which led to substantial improvements. Any remaining errors are ours. We acknowledge a grant $($ no. 30,649$)$ from the British Academy and the second author is grateful for the financial support by Spanish Ministry of Sciences and Technology, Project No. BEC20020309 .
}

** T.H. Kim: School of Economics, University of Nottingham; C. Muller: Dpto. Fundamentos de Análisis Económico, Universidad de Alicante. 


\title{
TWO-STAGE QUANTILE REGRESSION WHEN THE FIRST STAGE IS BASED ON QUANTILE REGRESSION
}

\author{
Tae-Hwan Kim and Christophe Muller
}

\begin{abstract}
We present the asymptotic properties of double-stage quantile regression estimators with random regressors, where the first stage is based on quantile regressions with the same quantile as in the second stage, which ensures robustness of the estimation procedure. We derive invariance properties with respect to the reformulation of the dependent variable. We propose a consistent estimator of the variance-covariance matrix of the new estimator. Finally, we investigate finite sample properties of this estimator by using Monte Carlo simulations.
\end{abstract}

Keywords: Two-stage estimation, Quantile regression, Endogeneity. 


\section{INTRODUCTION}

Quantile regressions are often used for wage and living standard analyses ${ }^{1}$, but also for studies of firm data and financial data. Quantile regressions produce robust estimates, particularly for misspecification errors related to non-normality and to the presence of outliers. They also help the researcher to focus her study on specific parts of the conditional distribution.

The researchers often study relations in which some right-hand-side variables are endogenous. For example, socio-economic variables, such as the education of the individual, appearing in wage equations may be endogenous. Other sources of endogeneity such as measurement errors are common.

When there are endogenous variables, the estimator of the parameter of interest is generally inconsistent. A well-known solution is the two-stage least squares (2SLS) estimation method in which one replaces the endogenous explanatory variables with their predictions from ancillary equations based on other exogenous variables. However, if researchers are interested in a specific part of the distribution of the structural variable, other than the mean, or if they want robust estimates, the 2SLS estimation method is not appropriate. Amemiya (1982) and Powell (1983) deal with the case of the double-stage least-absolute deviations (DSLAD) with fixed regressors, which allow researchers to focus on the median of the distribution of interest. In this paper, we extend Amemiya and Powell works by using quantile regressions and random exogenous variables. We use the same quantile estimation in the two steps and the resulting estimator of the structural parameter is termed "Double-Stage Quantile Regression estimator (DSQR)."

Other researchers have treated some endogeneity problems in quantile regressions. Chen and Portnoy (1996) study two-stage quantile regressions with symmetric error terms where the firststage estimators are trimmed least-squares estimators and LAD estimators. Other authors do not rely on the simple two-stage parametric approach favoured by many empirical economists. Kemp (1999) and Sakata (2001) study least absolute error difference estimators (LAED) for estimating a single equation from a simultaneous equation model ${ }^{2}$. Abadie et al. (2002) design a quantile treatment effects estimator, which is the solution to a convex programming problem with firststep nonparametric estimation of a nuisance function ${ }^{3}$. MaCurdy and Timmins (2000) propose an estimator for ARMA models adapted to the quantile regression framework. ${ }^{4}$

We do not follow these various approaches in this paper and we rather focus on two-stage estimation procedures familiar to empirical economists. Some empirical economists ${ }^{5}$ adopt a direct approach with a first stage of LS estimators. However, this approach may be delicate for the general type of problem that we consider since using LS estimation in the first step combined with Amemiya's reformulation of the dependent variable may produce an asymptotic bias (as will be shown in Appendix A). It can also destroy the robustness properties of the quantile regressions. We shall focus on the case when the first stage is composed of quantile regressions for which the quantile is the same as for the second stage.

\footnotetext{
${ }^{1}$ Buchinsky (1995), Machado and Mata (2001).

${ }^{2}$ These estimators are not based on first stage estimates of the reduced-form equations for the righthand-side endogenous variables. Instead, they use encompassed optimisation procedures, in which explicit concentration of formulae is impossible. The LAED optimisation is not a linear programming problem and a grid search must be used for the concentration.

${ }^{3}$ They deal with the case of binary treatment related to sample selectivity by modifying the typical objective function of the quantile regression problem with nonparametrically estimated weights.

${ }^{4} \mathrm{It}$ is based on the smoothing of conditional quantile conditions which are incorporated in a GMM framework.

${ }^{5}$ Arias et al. (2001), Garcia et al. (2001).
} 
The paper is organised as follows. Section 2 discusses the model and the assumptions. At this occasion we derive invariance properties with respect to the reformulation of the dependent variable. In Section 3 we prove the asymptotic normality of the DSQR and discuss the estimation of the asymptotic variance-covariance matrix. We emphasize that the same quantile should be used to estimate the reduced and structural forms in order to avoid asymptotic biases for all values of $q$. We present simulation results in Section 4. Finally, Section 5 concludes. The proofs are presented in Appendix B.

\section{THE MODEL}

We are interested in the structural parameter, $\alpha_{0}=\left(\gamma_{0}^{\prime}, \beta_{0}^{\prime}\right)^{\prime}$, in an equation in the following matrix form for a sample of $T$ observations:

$$
y=Y \gamma_{0}+X_{1} \beta_{0}+u
$$

where $[y, Y]$ is a $T \times(G+1)$ matrix of endogenous variables, $X_{1}$ is $T \times K_{1}$ matrix of exogenous variables and $u$ is a $T \times 1$ vector. The matrix $X_{2}$ contains $K_{2}\left(=K-K_{1}\right)$ exogenous variables absent from (1). In this situation the endogeneity of $Y$ in (1) may cause that $Q_{\theta}(u \mid Y) \neq Q_{\theta}(u)$, where $Q_{\theta}(\cdot)$ is the quantile function of order $\theta$, and $Q_{\theta}(\cdot \mid Y)$ is the quantile function of order $\theta$ conditional on $Y$. This non-equality can be used as a definition of endogeneity for quantile regressions. Moreover, we assume that $Y$ has the following reduced-form representation:

$$
Y=X \Pi_{0}+V
$$

where $X=\left[X_{1}, X_{2}\right]$ is a $T \times K$ matrix, $\Pi_{0}$ is a $K \times G$ matrix of unknown parameters and $V$ is a $T \times G$ matrix of unknown error terms. Then, the reduced-form representation of $y$ is:

$$
y=X \pi_{0}+v
$$

where $\pi_{0}=\left[\Pi_{0},\left(\begin{array}{c}I_{K_{1}} \\ 0\end{array}\right)\right] \alpha_{0}=H\left(\Pi_{0}\right) \alpha_{0}$ and $v=u+V \gamma_{0}$.

Define $\rho_{\theta}: R \rightarrow R^{+}$for given $\theta \in(0,1)$ as $\rho_{\theta}(z)=z \psi_{\theta}(z)$, where $\psi_{\theta}(z)=\theta-1_{[z \leq 0]}$ and $1_{[\cdot]}$ is the Kronecker index. As a natural extension of Amemiya (1982) and Powell (1983), we define the Double-Stage Quantile Regression estimator $(\operatorname{DSQR}(\theta, q)) \hat{\alpha}=\left(\hat{\gamma}^{\prime}, \hat{\beta}^{\prime}\right)^{\prime}$ of $\alpha_{0}$ as a solution to the following minimisation programme:

$$
\min _{\alpha} S_{T}(\alpha, \hat{\pi}, \hat{\Pi}, q, \theta)=\sum_{t=1}^{T} \rho_{\theta}\left(q y_{t}+(1-q) x_{t}^{\prime} \hat{\pi}-x_{t}^{\prime} H(\hat{\Pi}) \alpha\right)
$$

where $y_{t}$ and $x_{t}^{\prime}$ are the $t^{t h}$ elements in $y$ and $X$ respectively, $q$ is a positive constant chosen in advance by the researcher, and $\hat{\pi}$ and $\hat{\Pi}_{j}$ (the $j^{\text {th }}$ column of $\hat{\Pi}$ ) are the first stage estimators obtained by:

$$
\min _{\pi} \sum_{t=1}^{T} \rho_{\theta}\left(y_{t}-x_{t}^{\prime} \pi\right) \operatorname{andmin} \sum_{\Pi_{j}} \sum_{t=1}^{T} \rho_{\theta}\left(Y_{j t}-x_{t}^{\prime} \Pi_{j}\right),(j=1, \ldots, G),
$$

where $\pi$ and $\Pi_{j}$ are $K \times 1$ vectors and $Y_{j t}$ is the $(j, t)^{t h}$ element of $Y$. The reformulation of the dependent variable as $q y_{t}+(1-q) x_{t}^{\prime} \hat{\pi}$ has been introduced by Amemiya as a generalisation of a property of 2SLS, and an attempt to improve efficiency. Although, the ability of choosing the value of $q$ should yield estimators depending on this value, we show in the next proposition that there exist cases where the $\operatorname{DSQR}(\theta, q)$ is invariant with respect to the value of $q$. 
Proposition 1 Let $\delta(\theta, y, X)$ denotes the quantile regression estimator associated with quantile $\theta$, dependent variable $y$ and matrix of right-hand-side variables $X$. Moreover, let $\tilde{\alpha}=\delta(\theta, y, X H(\hat{\Pi}))$. Then, we have

(i) $\delta(\theta, q y+(1-q) X H(\hat{\Pi}) \tilde{\alpha}, X H(\hat{\Pi}))=\tilde{\alpha}$.

(ii) If $K_{2}=G$ and $H(\hat{\Pi})$ is of full column rank, then the $D S Q R(\theta, q)$ is given by $\hat{\alpha}=H(\hat{\Pi})^{-1} \hat{\pi}$.

The first result (i) is an invariance property of $\hat{\alpha}$ that is also verified by least square estimators. Although it does not correspond to the composite dependent variable that we shall consider later on, this result implies that iterating the estimation by changing the dependent variable in that way is useless, since the initial $\operatorname{DSQR}(\theta, 1)$ is obtained. The second property (ii) isolates the case of exact identification, where the distribution of the estimator, and in fact the estimator itself, do not depend on $q$. The argument and the direct calculus here are analogous to that used for Indirect Least-Squares estimator (ILS). Result (ii) shows how easily the $\operatorname{DSQR}(\theta, q)$ can be obtained when the exact identification condition is satisfied. In that case, no numerical minimisation of (4) is necessary and this is the precisely same way in which the ILS is obtained.

The following conditions will be useful to obtain the asymptotic representation of the $\operatorname{DSQR}(\theta, q)$, which we discuss in Appendix A, and the asymptotic normality of the $\operatorname{DSQR}(\theta, q)$.

Assumption 1 The sequence $\left\{\left(v_{t}, V_{t}, x_{t}\right)\right\}$ is independent and identically distributed (i.i.d.) where $u_{t}$ and $V_{t}$ are the $t^{\text {th }}$ elements in $u$ and $V$ respectively.

Assumption 2 (i) $E\left(\left\|x_{t}\right\|^{3}\right)<\infty$.

(ii) $H\left(\Pi_{0}\right)$ is of full column rank.

(iii) The conditional densities $f(\cdot \mid x)$ and $g_{j}(\cdot \mid x)$, respectively for $v_{t}$ and $V_{j t}$, are Lipschitz continuous for all $x$. Moreover, $Q_{0}=E\left\{f\left(0 \mid x_{t}\right) x_{t} x_{t}^{\prime}\right\}$ and $Q_{j}=E\left\{g_{j}\left(0 \mid x_{t}\right) x_{t} x_{t}^{\prime}\right\}$ are finite and positive definite.

(iv) $E\left\{\psi_{\theta}\left(v_{t}\right) \mid x_{t}\right\}=0$ and $E\left\{\psi_{\theta}\left(V_{j t}\right) \mid x_{t}\right\}=0(j=1, \ldots, G)$.

Assumption 2(i) is needed to generalise the stochastic equicontinuity result in Powell (1983). Assumptions 2(ii) and 2(iii) are standard in the literature. Assumption 2(iv) is a generalisation of Powell's assumption and states that zero is the quantile of order $\theta$ of $v_{t}$ and $V_{j t}$ conditionally on $x_{t}$. Assumption 2(iv) normalises the intercept on the $\theta^{t h}$ quantile of the distributions of $v_{t}$ and $V_{j t}$. The occurrence of a bias when different quantiles are used for the two stages, or when least-square estimators are used for the first stage, is discussed in Appendix A. For $q \neq 1$, distinct methods for first and second stages generally imply incompatible restrictions on error terms. We are now ready to study the asymptotic normality of the $\operatorname{DSQR}(\theta, q)$.

\section{ASYMPTOTIC NORMALITY AND COVARIANCE MATRIX}

The asymptotic normality of the $\operatorname{DSQR}(\theta, q)$ is based on its asymptotic representation derived in Appendix A. It is easy to see from this representation in (15) that the $\operatorname{DSQR}(\theta, q)$ is asymptotically robust because its influence function is a linear combination of bounded functions. The robustness would be lost if non-robust first-stage estimators were used.

Proposition 2 Under Assumptions 1 and $2, T^{1 / 2}\left(\hat{\alpha}-\alpha_{0}\right) \stackrel{d}{\rightarrow} N\left(0, D \Omega D^{\prime}\right)$, where $D=Q_{z z}^{-1} H\left(\Pi_{0}\right)^{\prime}$ $\left[I,-Q_{0} Q_{1}^{-1} \gamma_{01}, \ldots,-Q_{0} Q_{G}^{-1} \gamma_{0 G}\right], Q_{z z}=H\left(\Pi_{0}\right)^{\prime} Q_{0} H\left(\Pi_{0}\right)$ and $\Omega=E\left(\Sigma \otimes x_{t} x_{t}^{\prime}\right)$, where $\Sigma$ is the matrix of general term $\psi_{\theta}\left(W_{i t}\right) \psi_{\theta}\left(W_{j t}\right)$ with $W_{1 t}=v_{t}, W_{i t}=V_{i-1, t}$ for $2 \leq i \leq G+1$. 
Note that although the definition of $\hat{\alpha}$ depends on $q$, its asymptotic law does not. In that case the first-stage estimator $\hat{\pi}$ intervenes in the calculation of $\hat{\alpha}$, but not in its asymptotic distribution. However, $\hat{\pi}$ can still be used for a consistent estimator of the asymptotic covariance matrix. In order to conduct inference based on $\hat{\alpha}$, it is necessary to have consistent estimators of $D$ and $\Omega$. We now develop such estimators by using the plug-in principle.

Assumption 3 (i) There exist positive constants $f_{0}$ and $g_{0 j}$ such that $f(\cdot \mid x) \leq f_{0}$ and $g_{j}(\cdot \mid x) \leq g_{0 j}$ for all $x$.

(ii) There exists a stochastic sequence $\left\{\hat{c}_{j T}\right\}$ and a non-stochastic sequence $\left\{c_{j T}\right\}$ for $j=0,1, \ldots, G$ such that we have $\hat{c}_{j T} / c_{j T} \stackrel{p}{\rightarrow} 1, c_{j T}=o_{p}(1)$ and $c_{j T}^{-1}=o_{p}\left(T^{1 / 2}\right)$.

(iii) $E\left(\left\|x_{t}\right\|^{4}\right)<\infty$.

We defined our covariance estimators as follows:

$$
\hat{D}=\hat{Q}_{z z}^{-1} H(\hat{\Pi})^{\prime}\left[I,-\hat{Q}_{0} \hat{Q}_{1}^{-1} \hat{\gamma}_{1}, \ldots,-\hat{Q}_{0} \hat{Q}_{G}^{-1} \hat{\gamma}_{G}\right]
$$

where $\hat{Q}_{z z}=H(\hat{\Pi})^{\prime} \hat{Q}_{0} H(\hat{\Pi}), \hat{Q}_{0}=\left(2 \hat{c}_{0 T} T\right)^{-1} \sum_{t=1}^{T} 1_{\left[-\hat{c}_{0 T} \leq \hat{v}_{t} \leq \hat{c}_{0 T}\right]} x_{t} x_{t}^{\prime}, \hat{Q}_{j}=\left(2 \hat{c}_{j T} T\right)^{-1} \sum_{t=1}^{T}$ $1_{\left[-\hat{c}_{j T} \leq \hat{V}_{j t} \leq \hat{c}_{j T}\right]} x_{t} x_{t}^{\prime}, \hat{v}_{t}=y_{t}-x_{t}^{\prime} \hat{\pi}$ and $\hat{V}_{j t}=Y_{j t}-x_{t}^{\prime} \hat{\Pi}(j=1, \ldots, G)$;

$$
\hat{\Omega}=T^{-1} \sum_{t=1}^{T} \hat{\Sigma}_{t} \otimes x_{t} x_{t}^{\prime},
$$

where $\hat{\Sigma}_{t}=\hat{W} \otimes x_{t} x_{t}^{\prime}$ and $\hat{W}$ is the matrix of general term $\psi_{\theta}\left(\hat{W}_{i t}\right) \psi_{\theta}\left(\hat{W}_{j t}\right)$ with $\hat{W}_{1 t}=\hat{v}_{t}, \hat{W}_{i t}=$ $\hat{V}_{i-1, t}$ for $2 \leq i \leq G+1$. Then, our proposed covariance matrix estimator is $\hat{D} \hat{\Omega} \hat{D}^{\prime}$.

Proposition 3 Under Assumptions 1,2 and 3, $\hat{D} \stackrel{p}{\rightarrow} D$ and $\hat{\Omega} \stackrel{p}{\rightarrow} \Omega$.

To the best of our knowledge that is the first time that a proof of convergence of the variancecovariance matrix of the two-stage quantile estimators is given and even with non-random regressors. We now complete these asymptotic results with small sample simulations.

\section{MONTE CARLO SIMULATIONS}

In this section, we conduct simulation experiments to investigate the finite-sample properties of the $\operatorname{DSQR}(\theta, q)^{6}$, notably in terms of the endogeneity and robustness problems.

The data generating process used in the simulations is described in Appendix $\mathrm{C}$, which shows that the equation of interest is over-identified and the parameter values are $\gamma_{0}=0.5$ and $\beta_{0}^{\prime}=$ $(1,0.2)$. We generate the error terms by using three alternative distributions: the standard normal $\mathrm{N}(0,1)$, the Student- $t$ with 3 degrees of freedom $t(3)$ and the Lognormal $\operatorname{LN}(0,1)$. The exogenous variables $x_{t}$ are also drawn in a normal distribution at each replication. The number of replications is 1,000 . For each replication, we estimate the parameter values $\gamma_{0}$ and $\beta_{0}$ and the deviation of the estimates from the true values. Then, we compute the sample mean and sample standard deviation (when useful, the sample median and the sample interquartile range) over the 1,000 replications.

The performance of the one-stage quantile regression estimator for the different distributions is displayed in Table 1 for the $\mathrm{N}(0,1)$ case. The results for the other distributions are qualitatively similar and are not reported. This estimator exhibits a systematic bias in finite samples, which does not disappear as the sample size increases from 50 to 300 .

\footnotetext{
${ }^{6}$ See also Ribeiro (1998) for small sample simulations of two-stage LAD estimation with a first step of LS or LAD estimators.
} 
Table 1. Simulation Means and Standard Deviations of the Deviations from the True Value with One Step Quantile Estimator: $\mathbf{N}(\mathbf{0 , 1})$

\begin{tabular}{|c|c|c|c|c|c|c|c|}
\hline & & $\theta$ & 0.05 & 0.25 & 0.50 & 0.75 & 0.95 \\
\hline \multirow{7}{*}{$T=50$} & \multirow{3}{*}{$\hat{\gamma}$} & & & & & & \\
\hline & & Mean & -0.45 & -0.43 & -0.43 & -0.44 & -0.44 \\
\hline & & Std & 0.29 & 0.18 & 0.16 & 0.18 & 0.29 \\
\hline & \multirow[t]{2}{*}{$\hat{\beta}_{0}$} & Mean & 1.30 & 1.31 & 1.38 & 1.44 & 1.52 \\
\hline & & Std & 1.40 & 0.74 & 0.55 & 0.50 & 0.56 \\
\hline & \multirow[t]{2}{*}{$\hat{\beta}_{1}$} & Mean & 0.17 & 0.17 & 0.16 & 0.16 & 0.16 \\
\hline & & Std & 0.35 & 0.22 & 0.20 & 0.22 & 0.34 \\
\hline \multirow{6}{*}{$T=300$} & \multirow{2}{*}{$\hat{\gamma}$} & & & & & & \\
\hline & & $\begin{array}{r}\text { Mean } \\
\text { Std }\end{array}$ & -0.44 & $\begin{array}{r}-0.44 \\
0.07\end{array}$ & $\begin{array}{r}-0.44 \\
0.06\end{array}$ & $\begin{array}{r}-0.44 \\
0.07\end{array}$ & $\begin{array}{r}-0.44 \\
0.11\end{array}$ \\
\hline & \multirow[t]{2}{*}{$\hat{\beta}_{0}$} & Mean & 1.23 & 1.32 & 1.39 & 1.46 & 1.57 \\
\hline & & Std & 0.54 & 0.28 & 0.22 & 0.20 & 0.22 \\
\hline & \multirow[t]{2}{*}{$\hat{\beta}_{1}$} & Mean & 0.17 & 0.16 & 0.16 & 0.17 & 0.16 \\
\hline & & Std & 0.13 & 0.09 & 0.08 & 0.09 & 0.13 \\
\hline
\end{tabular}

Table 2. Simulation Means and Standard Deviations of the Deviations from the True Value with One Step Quantile Estimator: $t$ (3)

\begin{tabular}{|c|c|c|c|c|c|c|c|}
\hline & & $\theta$ & 0.05 & 0.25 & 0.50 & 0.75 & 0.95 \\
\hline \multirow{6}{*}{$T=50$} & \multirow[t]{2}{*}{$\hat{\gamma}$} & Mean & -0.58 & -0.49 & -0.48 & -0.50 & -0.59 \\
\hline & & Std & 0.44 & 0.16 & 0.13 & 0.16 & 0.43 \\
\hline & \multirow[t]{2}{*}{$\hat{\beta}_{0}$} & Mean & 1.89 & 1.53 & 1.55 & 1.64 & 1.89 \\
\hline & & Std & 2.34 & 0.65 & 0.48 & 0.48 & 1.23 \\
\hline & \multirow[t]{2}{*}{$\hat{\beta}_{1}$} & Mean & 0.20 & 0.18 & 0.18 & 0.19 & 0.20 \\
\hline & & Std & 0.78 & 0.27 & 0.22 & 0.26 & 0.73 \\
\hline & & & & & & & \\
\hline \multirow{6}{*}{$T=300$} & \multirow[t]{2}{*}{$\hat{\gamma}$} & Mean & -0.57 & -0.50 & -0.49 & -0.50 & -0.57 \\
\hline & & Std & 0.15 & 0.06 & 0.05 & 0.06 & 0.15 \\
\hline & \multirow[t]{2}{*}{$\hat{\beta}_{0}$} & Mean & 1.92 & 1.56 & 1.57 & 1.64 & 1.74 \\
\hline & & Std & 0.84 & 0.26 & 0.18 & 0.19 & 0.37 \\
\hline & \multirow[t]{2}{*}{$\hat{\beta}_{1}$} & Mean & 0.22 & 0.18 & 0.18 & 0.18 & 0.21 \\
\hline & & Std & 0.28 & 0.10 & 0.08 & 0.11 & 0.27 \\
\hline
\end{tabular}

Table 3. Simulation Means and Standard Deviations of the Deviations from the True Value with One Step Quantile Estimator: $\mathbf{L N}(0,1)$

\begin{tabular}{|c|c|c|c|c|c|c|c|}
\hline & & $\theta$ & 0.05 & 0.25 & 0.50 & 0.75 & 0.95 \\
\hline \multirow{6}{*}{$T=50$} & \multirow{2}{*}{$\hat{\gamma}$} & $T_{-}$ & 020 & 0.47 & 059 & 054 & 056 \\
\hline & & $\begin{array}{r}\text { Mean } \\
\text { Std }\end{array}$ & $\begin{array}{l}0.38 \\
0.15\end{array}$ & $\begin{array}{l}0.47 \\
0.09\end{array}$ & $\begin{array}{l}0.52 \\
0.07\end{array}$ & $\begin{array}{l}0.34 \\
0.05\end{array}$ & $\begin{array}{l}0.06 \\
0.05\end{array}$ \\
\hline & \multirow[t]{2}{*}{$\hat{\beta}_{0}$} & Mean & -0.34 & -0.18 & 0.02 & 0.29 & 0.81 \\
\hline & & Std & 0.18 & 0.09 & 0.07 & 0.10 & 0.25 \\
\hline & \multirow[t]{2}{*}{$\hat{\beta}_{1}$} & Mean & -0.14 & -0.17 & -0.19 & -0.20 & -0.21 \\
\hline & & Std & 0.10 & 0.07 & 0.07 & 0.08 & 0.12 \\
\hline \multirow{6}{*}{$T=300$} & \multirow[t]{2}{*}{$\hat{\gamma}$} & Mean & 0.35 & 0.48 & 0.53 & 0.56 & 0.58 \\
\hline & & Std & 0.08 & 0.04 & 0.03 & 0.02 & 0.02 \\
\hline & \multirow[t]{2}{*}{$\hat{\beta}_{0}$} & Mean & -0.31 & -0.18 & 0.02 & 0.31 & 0.92 \\
\hline & & Std & 0.09 & 0.04 & 0.03 & 0.04 & 0.10 \\
\hline & \multirow[t]{2}{*}{$\hat{\beta}_{1}$} & Mean & -0.13 & -0.18 & -0.19 & -0.21 & -0.21 \\
\hline & & Std & 0.04 & 0.03 & 0.03 & 0.03 & 0.05 \\
\hline
\end{tabular}


The results for the $\operatorname{DSQR}(\theta, q)$ with $\mathrm{N}(0,1)$ are provided in Tables 2(a) and 2(b). We select two illustrative values ( 0.5 and 1$)$ for $q$. As shown in Proposition 1 , when the system is exactly identified, this dependence on $q$ does not exist, which is confirmed by our simulations of this case (not shown). Whereas the $\operatorname{DSQR}(\theta, q)$ does not depend on $q$ asymptotically, it does in finite samples, but as we increase the sample size to 300 , the results for different $q$ 's become quantitatively similar. The means of the $\operatorname{DSQR}(\theta, q)$ estimates, $\left(\hat{\gamma}-\gamma_{0}, \hat{\beta}-\beta_{0}\right)$, are much closer to zero than the means of the one-step quantile estimator over all values of $\theta$, although the corresponding standard deviations are generally greater.

For other distributions too, the changes in the value of $q$ do not substantially modify the results. Hence, for these two distributions, $t(3)$ and $\operatorname{LN}(0,1)$, we report only the case $q=1$. The results with $t(3)$, which are in Table 3 show similar features. However, the fatter tails of the errors entail accuracy losses for both one-stage and two-stage estimators. The results with lognormal error terms, shown in Table 4, differ in that both estimators are severely biased for large quantiles (for $\theta=0.95$ ). The bias of the $\operatorname{DSQR}(\theta, q)$ diminishes when the sample size rises to $T=300$. In a simulation available upon request, the bias disappears for a sufficiently large number of observations, as opposed to the case of the one-stage estimator. Also, the performance of the $\operatorname{DSQR}(\theta, q)$ for the lognormal case is the best for small quantiles $(\theta=0.05)$, in contrast with the usual better performance of the $\operatorname{DSQR}(\theta, q)$ for quantiles around $\theta=0.5$ and symmetric distributions. The formula of the diagonal term of the covariance matrix $D \Omega D^{\prime}$ suggests us a conjecture for the occurrence of such effect in large samples, which may extend to small samples in some cases. Indeed, because of the asymmetric shape of the lognormal distribution, $f\left(0 \mid x_{t}\right)$ and $g_{j}\left(0 \mid x_{t}\right)$ for large values of $\theta$ when the $\theta^{\text {th }}$ quantile is zero can be very close to zero. This inflates the roles of some terms in the diagonal terms of $D \Omega D^{\prime}$, which may lead to a large variance for the $\operatorname{DSQR}(\theta, q)$ and cause the large finite-sample bias for $\theta=0.95$ and $\mathrm{LN}(0,1)$.

For all the types of error terms, the one-stage estimator is severely biased. In contrast, the $\operatorname{DSQR}(\theta, q)$ has good finite sample properties, although a too small sample size or too extreme quantiles $(\theta=0.05,0.95)$ may degrade its performance. Sample medians and sample interquartile ranges have been calculated to supplement sample means and sample standard deviations respectively. We have not found any significant difference between the robust measures and the usual measures, except in the case where the error terms are drawn from $\operatorname{LN}(0,1)$ and with large quantiles $(\theta=0.95)$. In that case only, the robust measures are reported in the brackets, next to their corresponding usual measures in Table 4. The dispersion of the sampling distribution of the deviations is smaller when robust measures are used.

One of the justifications for using the $\operatorname{DSQR}(\theta, q)$ is that it is resistant to outliers. To confirm this property, we conduct a separate Monte Carlo experiment in which we compare the $\operatorname{DSQR}(\theta, q)$ with the 2SLS when one outlier occurs for the dependent variable $y_{t}$. Following Cowell and Flachaire (2002), the outlier is the randomly selected observation of $y_{t}$ and multiplied by 15 . The results in the normal error case are reported in Table 5 for the 2SLS, which is invariant to the value of $q$, and for the $\operatorname{DSQR}(\theta, 1)$. Other distributions and the other values of $q$ deliver similar results. The results show that the $\operatorname{DSQR}(\theta, q)$ is much more robust to outliers than the 2SLS, which is still more obvious when medians and interquartiles indicators are used.

\section{CONCLUSION}

We study in this paper the asymptotic properties, the invariance, the robustness and the finite sample properties of double-stage quantile regression estimators with first-stage quantile regressions defined on the same quantile as the second stage. Our results permit valid inferences in models estimated using quantile regressions with random regressors, in which the possible endogeneity of some explanatory variables is treated via preliminary predictive quantile regressions. 
Table 4(a). Simulation Means and Standard Deviations of the Deviations from the True Value with $\operatorname{DS} Q R(\theta, q=0.1): \mathbf{N}(\mathbf{0}, \mathbf{1})$.

\begin{tabular}{|c|c|c|c|c|c|c|c|}
\hline & & $\theta$ & 0.05 & 0.25 & 0.50 & 0.75 & 0.95 \\
\hline \multirow{6}{*}{$T=50$} & \multirow[t]{2}{*}{$\hat{\gamma}$} & Mean & -0.06 & 0.01 & 0.01 & 0.01 & -0.03 \\
\hline & & Std & 0.80 & 0.64 & 0.40 & 0.48 & 0.93 \\
\hline & \multirow[t]{2}{*}{$\hat{\beta}_{0}$} & Mean & -0.07 & -0.11 & -0.02 & 0.04 & 0.37 \\
\hline & & Std & 2.65 & 2.08 & 1.26 & 1.55 & 3.05 \\
\hline & \multirow[t]{2}{*}{$\hat{\beta}_{1}$} & Mean & 0.03 & 0.01 & 0.00 & -0.01 & 0.03 \\
\hline & & Std & 0.52 & 0.30 & 0.26 & 0.30 & 0.50 \\
\hline & & & & & & & \\
\hline \multirow{6}{*}{$T=300$} & \multirow[t]{2}{*}{$\hat{\gamma}$} & Mean & 0.01 & 0.01 & 0.01 & 0.01 & 0.00 \\
\hline & & Std & 0.24 & 0.15 & 0.14 & 0.15 & 0.23 \\
\hline & \multirow[t]{2}{*}{$\hat{\beta}_{0}$} & Mean & -0.11 & -0.03 & -0.02 & 0.00 & 0.07 \\
\hline & & Std & 0.80 & 0.48 & 0.45 & 0.48 & 0.76 \\
\hline & \multirow[t]{2}{*}{$\hat{\beta}_{1}$} & Mean & 0.00 & 0.00 & 0.00 & 0.00 & 0.00 \\
\hline & & Std & 0.17 & 0.10 & 0.10 & 0.11 & 0.16 \\
\hline
\end{tabular}

Table 4(b). Simulation Means and Standard Deviations of the Deviations from the True Value with $\operatorname{DS} Q R(\theta, q=0.5): \mathbf{N}(\mathbf{0}, \mathbf{1})$.

\begin{tabular}{|c|c|c|c|c|c|c|c|}
\hline & & $\theta$ & 0.05 & 0.25 & 0.50 & 0.75 & 0.95 \\
\hline \multirow{7}{*}{$T=50$} & \multirow{3}{*}{$\hat{\gamma}$} & & & & & & \\
\hline & & Mean & -0.07 & 0.01 & 0.00 & 0.01 & -0.03 \\
\hline & & Std & 0.78 & 0.64 & 0.40 & 0.48 & 0.97 \\
\hline & \multirow[t]{2}{*}{$\hat{\beta}_{0}$} & Mean & 0.15 & -0.07 & 0.00 & -0.02 & 0.14 \\
\hline & & Std & 2.58 & 2.10 & 1.26 & 1.54 & 3.18 \\
\hline & \multirow[t]{2}{*}{$\hat{\beta}_{1}$} & Mean & 0.04 & 0.01 & 0.00 & 0.00 & 0.03 \\
\hline & & Std & 0.50 & 0.29 & 0.26 & 0.30 & 0.50 \\
\hline \multirow{6}{*}{$T=300$} & \multirow[t]{2}{*}{$\hat{\gamma}$} & Mean & 0.01 & 0.01 & 0.01 & 0.01 & 0.00 \\
\hline & & Std & 0.25 & 0.15 & 0.14 & 0.15 & 0.23 \\
\hline & \multirow[t]{2}{*}{$\hat{\beta}_{0}$} & Mean & -0.04 & -0.02 & -0.02 & -0.02 & 0.01 \\
\hline & & Std & 0.80 & 0.48 & 0.45 & 0.48 & 0.74 \\
\hline & \multirow[t]{2}{*}{$\hat{\beta}_{1}$} & Mean & -0.01 & 0.00 & 0.00 & 0.00 & 0.00 \\
\hline & & Std & 0.17 & 0.10 & 0.10 & 0.11 & 0.16 \\
\hline
\end{tabular}

Table 4(c). Simulation Means and Standard Deviations of the Deviations from the True Value with $\operatorname{DSQR}(\theta, q=1): \mathbf{N}(\mathbf{0 , 1})$.

\begin{tabular}{|c|c|c|c|c|c|c|c|}
\hline & & $\theta$ & 0.05 & 0.25 & 0.50 & 0.75 & 0.95 \\
\hline \multirow{6}{*}{$T=50$} & \multirow[t]{2}{*}{$\hat{\gamma}$} & Mean & -0.04 & 0.02 & 0.00 & 0.00 & -0.04 \\
\hline & & Std & 0.85 & 0.68 & 0.41 & 0.49 & 0.88 \\
\hline & \multirow[t]{2}{*}{$\hat{\beta}_{0}$} & Mean & 0.11 & -0.08 & -0.01 & -0.02 & 0.11 \\
\hline & & Std & 2.82 & 2.22 & 1.30 & 1.59 & 2.87 \\
\hline & \multirow{2}{*}{$\hat{\beta}_{1}$} & Mean & 0.02 & 0.00 & 0.00 & 0.00 & 0.02 \\
\hline & & Std & 0.53 & 0.31 & 0.26 & 0.31 & 0.51 \\
\hline \multirow{6}{*}{$T=300$} & \multirow{2}{*}{$\hat{\gamma}$} & Mon & 001 & 001 & 001 & 000 & 000 \\
\hline & & Std & 0.25 & 0.15 & 0.14 & 0.15 & 0.23 \\
\hline & \multirow[t]{2}{*}{$\hat{\beta}_{0}$} & Mean & -0.03 & -0.02 & -0.02 & -0.02 & 0.01 \\
\hline & & Std & 0.81 & 0.49 & 0.46 & 0.50 & 0.75 \\
\hline & \multirow[t]{2}{*}{$\hat{\beta}_{1}$} & Mean & 0.00 & 0.00 & 0.00 & 0.00 & 0.00 \\
\hline & & Std & 0.18 & 0.10 & 0.10 & 0.11 & 0.16 \\
\hline
\end{tabular}


Table 5. Simulation Means and Standard Deviations of the Deviations from the True Value with $\operatorname{DSQR}(\theta, q=1): t(\mathbf{3})$.

\begin{tabular}{|c|c|c|c|c|c|c|c|}
\hline & & $\theta$ & 0.05 & 0.25 & 0.50 & 0.75 & 0.95 \\
\hline \multirow{6}{*}{$T=50$} & \multirow[t]{2}{*}{$\bar{\gamma}$} & Mean & -0.31 & -0.02 & 0.00 & -0.05 & -0.32 \\
\hline & & Std & 1.40 & 0.73 & 0.49 & 0.81 & 1.35 \\
\hline & \multirow[t]{2}{*}{$\hat{\beta}_{0}$} & Mean & 0.80 & 0.07 & 0.00 & 0.19 & 1.26 \\
\hline & & Std & 4.76 & 2.26 & 1.56 & 2.55 & 4.34 \\
\hline & \multirow[t]{2}{*}{$\hat{\beta}_{1}$} & Mean & 0.14 & 0.00 & 0.00 & 0.03 & 0.13 \\
\hline & & Std & 1.15 & 0.37 & 0.31 & 0.44 & 1.16 \\
\hline & & & & & & & \\
\hline \multirow{6}{*}{$T=300$} & \multirow[t]{2}{*}{$\hat{\gamma}$} & Mean & -0.06 & 0.00 & -0.01 & 0.00 & -0.07 \\
\hline & & Std & 0.76 & 0.19 & 0.15 & 0.19 & 0.63 \\
\hline & \multirow[t]{2}{*}{$\hat{\beta}_{0}$} & Mean & 0.14 & 0.00 & 0.04 & 0.02 & 0.23 \\
\hline & & Std & 2.46 & 0.62 & 0.49 & 0.62 & 2.17 \\
\hline & \multirow[t]{2}{*}{$\hat{\beta}_{1}$} & Mean & 0.03 & -0.01 & 0.00 & -0.01 & 0.02 \\
\hline & & Std & 0.41 & 0.13 & 0.11 & 0.14 & 0.41 \\
\hline
\end{tabular}

Table 6. Simulation Means and Standard Deviations of the Deviations from the True Value with $\operatorname{DS} Q R(\theta, q=1): \mathbf{L N}(\mathbf{0}, \mathbf{1})$.

\begin{tabular}{|c|c|c|c|c|c|c|c|}
\hline & & $\theta$ & 0.05 & 0.25 & 0.50 & 0.75 & 0.95 \\
\hline \multirow{6}{*}{$T=50$} & \multirow{2}{*}{$\hat{\gamma}$} & Mean & 000 & 000 & 002 & 012 & $049(0-47)$ \\
\hline & & Std & 0.08 & 0.11 & 0.34 & 0.70 & $0.44(0.34)$ \\
\hline & \multirow[t]{2}{*}{$\hat{\beta}_{0}$} & Mean & -0.06 & -0.05 & -0.02 & 0.00 & $0.37(0.40)$ \\
\hline & & Std & 0.05 & 0.07 & 0.18 & 0.31 & $0.78(0.60)$ \\
\hline & \multirow{2}{*}{$\hat{\beta}_{1}$} & Mean & 0.00 & 0.00 & -0.01 & -0.04 & $-0.19(-0.20)$ \\
\hline & & Std & 0.06 & 0.08 & 0.15 & 0.27 & $0.49(0.46)$ \\
\hline \multirow{6}{*}{$T=300$} & \multirow[t]{2}{*}{$\hat{\gamma}$} & Mean & 0.00 & 0.00 & 0.00 & 0.00 & $0.29(0.30)$ \\
\hline & & Std & 0.02 & 0.04 & 0.07 & 0.15 & $0.52(0.33)$ \\
\hline & \multirow[t]{2}{*}{$\hat{\beta}_{0}$} & Mean & -0.08 & -0.06 & -0.04 & 0.01 & $0.28(0.32)$ \\
\hline & & Std & 0.02 & 0.03 & 0.05 & 0.10 & $0.47(0.43)$ \\
\hline & \multirow[t]{2}{*}{$\hat{\beta}_{1}$} & Mean & 0.00 & 0.00 & 0.00 & 0.00 & $-0.10(-0.12)$ \\
\hline & & Std & 0.02 & 0.03 & 0.05 & 0.11 & $0.40(0.33)$ \\
\hline
\end{tabular}

Table 7. Simulation Means and Standard Deviations of the Deviations from the True Value with $\operatorname{DS} Q R(\theta, q=1)$ and $2 S L S$ with a single outlier: $\mathbf{N}(\mathbf{0 , 1})$.

\begin{tabular}{|c|c|c|c|c|c|c|c|c|}
\hline & & $\theta$ & 0.05 & 0.25 & 0.50 & 0.75 & 0.95 & $2 S L S$ \\
\hline \multirow{6}{*}{$T=50$} & \multirow{2}{*}{$\hat{\gamma}$} & Mean & -004 & 002 & 001 & 001 & $00 ?$ & 024 \\
\hline & & Std & 0.86 & 0.64 & 0.42 & 0.52 & 1.56 & 2.08 \\
\hline & \multirow[t]{2}{*}{$\hat{\beta}_{0}$} & Mean & 0.12 & -0.05 & 0.00 & 0.02 & 0.53 & 0.23 \\
\hline & & Std & 2.89 & 2.05 & 1.33 & 1.67 & 4.91 & 6.51 \\
\hline & \multirow[t]{2}{*}{$\hat{\beta}_{1}$} & Mean & 0.03 & 0.00 & 0.00 & 0.00 & 0.01 & -0.01 \\
\hline & & Std & 0.53 & 0.31 & 0.27 & 0.33 & 1.47 & 1.54 \\
\hline \multirow{6}{*}{$T=300$} & \multirow{2}{*}{$\hat{\gamma}$} & Mean & 0.01 & 0.01 & 0.01 & 0.00 & 0.00 & 0.04 \\
\hline & & Std & 0.25 & 0.15 & 0.14 & 0.15 & 0.24 & 0.32 \\
\hline & \multirow[t]{2}{*}{$\hat{\beta}_{0}$} & Mean & -0.03 & -0.02 & -0.02 & -0.01 & 0.03 & 0.04 \\
\hline & & Std & 0.81 & 0.49 & 0.46 & 0.50 & 0.79 & 1.03 \\
\hline & \multirow[t]{2}{*}{$\hat{\beta}_{1}$} & Mean & 0.00 & 0.00 & 0.00 & 0.00 & 0.01 & 0.01 \\
\hline & & Std & 0.18 & 0.11 & 0.10 & 0.11 & 0.17 & 0.25 \\
\hline
\end{tabular}




\section{References}

[1] Abadie, A., J. Angrist and G. Imbens (2002). Instrumental variables estimates of the effect of subsidized training on the quantiles of trainee earnings. Econometrica 70, 91-117.

[2] Amemiya, T. (1982). Two stage least absolute deviations estimators. Econometrica 50, 689711.

[3] Andrews, D.W.K. (1994). Empirical process methods in econometrics. Handbook of Econometrics, Volume IV, eds. R.F. Engle and D.L. McFadden, New York: North-Holland, pp. 2247-94.

[4] Arias, O., K.F. Hallock and W. Sosa-Escudero (2001). Individual heterogeneity in the returns to schooling: Instrumental variables quantile regression using twins data. Empirical Economics $26,7-40$.

[5] Buchinsky, M. (1995). Quantile regression, Box-Cox transformation model, and the U.S. wage structure, 1963-1987. Journal of Econometrics 65, 109-54.

[6] Chen, L.-A. and S. Portnoy (1996). Two-stage regression quantiles and two-stage trimmed least squares estimators for structural equation models. Commun. Statist.-Theory Meth. 25, 1005-32.

[7] Cowell, F. and E. Flachaire (2000). Sensitivity of inequality measures to extreme values. Mimeo London School of Economics.

[8] Garcia, J., P.J. Hernandez and A. Lopez-Nicolàs (2001). How wide is the gap? An investigation of gender wage differences using quantile regression. Empirical Economics 26, 149-67.

[9] Kemp, G. (1999). Least absolute error difference estimation of a single equation from a simultaneous equations system. Mimeo University of Essex.

[10] Kim, T. and C. Muller (2000). Two-stage quantile regression. Nottingham University Working Paper.

[11] Kim, T. and C. Muller (2003). Two-stage quantile regression when the first stage is based on quantile regression. Nottingham University Working Paper.

[12] Koenker, R. and Q. Zhao (1996). Conditional quantile estimation and inference for ARCH models. Econometric Theory 12, 793-813.

[13] Machado, J.A.F. and J. Mata (2001). Earning functions in Portugal 1982-1994: Evidence from quantile regressions. Empirical Economics 26, 115-34.

[14] MaCurdy, T. and C. Timmins (2000). Bounding the influence of attrition on intertemporal wage variation in the NLSY. Mimeo Stanford University.

[15] Pollard, D. (1991). Asymptotics for least absolute deviations regression estimators. Econometric Theory 7, 186-199. 
[16] Powell, J. (1983). The asymptotic normality of two-stage least absolute deviations estimators. Econometrica 51, 1569-75.

[17] Ribeiro, E. (1998). Small sample evidence of quantile regression for structural models: estimation and testing. Revista de Econometria 18, 215-44.

[18] Sakata, S. (2001). Instrumental variable estimation based on mean absolute deviation. Mimeo University of Michigan.

\section{A. APPENDIX. ASYMPTOTIC REPRESENTATION}

We define an empirical process $M_{T}^{*}(\zeta)$ by

$$
M_{T}^{*}(\zeta)=T^{-1 / 2} \sum_{t=1}^{T} x_{t} \psi_{\theta}\left(q v_{t}-x_{t}^{\prime} \zeta\right)=T^{-1 / 2} \sum_{t=1}^{T} m^{*}\left(w_{t}, \zeta\right),
$$

where $\zeta$ is a $K \times 1$ vector, $w_{t}=\left(v_{t}, x_{t}^{\prime}\right)^{\prime}, m^{*}\left(w_{t}, \zeta\right)=x_{t} \psi_{\theta}\left(q v_{t}-x_{t}^{\prime} \zeta\right)$ and $\zeta=T^{-1 / 2} \Delta \in R^{K}$ with the norm $\|$.$\| . Let V_{T}^{*}(\zeta)=T^{-1 / 2} \sum_{t=1}^{T}\left[m^{*}\left(w_{t}, \zeta\right)-E\left\{m^{*}\left(w_{t}, \zeta\right)\right\}\right]=M_{T}^{*}(\zeta)-E\left(M_{T}^{*}(\zeta)\right)$. Since the function $\psi_{\theta}$ is of bounded variations, Assumptions 1 and 2 are sufficient to apply Theorems 1-3 in Andrews (1994), which leads to:

$$
\sup _{\left\|\zeta_{1}-\zeta_{2}\right\| \leq L^{*}}\left\|V_{T}^{*}\left(\zeta_{1}\right)-V_{T}^{*}\left(\zeta_{2}\right)\right\|=o_{p}(1)
$$

for any finite and positive scalar $L^{*}$. We outline the proof below. In order to apply Theorem 1 in Andrews (1994), the following two conditions must be verified:

(i) $m^{*}\left(w_{t}, \zeta\right)$ satisfies Pollard's entropy condition with some envelop $\bar{M}\left(w_{t}\right)$;

(ii) For some $\delta>2, \lim _{T \rightarrow \infty} T^{-1} \sum_{t=1}^{T} E\left[\left\{\bar{M}\left(w_{t}\right)\right\}^{\delta}\right]<\infty$.

Let $f_{1}\left(w_{t}, \zeta\right)=x_{t}$ and $f_{2}\left(w_{t}, \zeta\right)=\psi_{\theta}\left(q v-x_{t}^{\prime} \zeta\right)$ so that $m^{*}\left(w_{t}, \zeta\right)=f_{1}\left(w_{t}, \zeta\right) f_{2}\left(w_{t}, \zeta\right)$. Since $f_{1}(\cdot, \zeta)$ and $f_{2}(\cdot, \zeta)$ are Type I functions with envelopes $\left\|x_{t}\right\|$ and 1 respectively (see Andrews, 1994, for definitions $), m^{*}\left(w_{t}, \zeta\right)$ satisfies Pollard's entropy condition with envelope $\max \left(1,\left\|x_{t}\right\|\right)$ because it is a product of two Type I functions (See Theorems 2 and 3 in Andrews, 1994). Hence, (i) is verified. The second condition is now given by $\lim _{T \rightarrow \infty} T^{-1} \sum_{t=1}^{T} E\left[\left\{\bar{M}\left(w_{t}\right)\right\}^{\delta}\right]=E\left[\left\{\max \left(1,\left\|x_{t}\right\|\right)\right\}^{\delta}\right]$ which is bounded by Assumption 2(i). Hence, the result in (6) now follows.

For any $\zeta_{1}, \zeta_{2}$ in $R^{K}$ and $L^{*}(>0)$ in $R$, we can find $\Delta_{1}, \Delta_{2}$ in $R^{K}$ and $L(>0)$ in $R$ such that $\zeta_{1}=T^{-1 / 2} \Delta_{1}, \zeta_{2}=T^{-1 / 2} \Delta_{2}$ and $L^{*}=T^{-1 / 2} L$. With these notations, we define the following functions: $V_{T}(\Delta)=M_{T}(\Delta)-E\left(M_{T}(\Delta)\right)$ where $M_{T}(\Delta)=T^{-1 / 2} \sum_{t=1}^{T} m\left(w_{t}, \Delta\right)$ and $m\left(w_{t}, \Delta\right)=$ $x_{t} \psi_{\theta}\left(q v_{t}-T^{-1 / 2} x_{t}^{\prime} \Delta\right)$. Then, it is easy to see that the result in (6) can be written as

$$
\sup _{\left\|\Delta_{1}-\Delta_{2}\right\| \leq L}\left\|V_{T}\left(\Delta_{1}\right)-V_{T}\left(\Delta_{2}\right)\right\|=o_{p}(1) .
$$

By setting $\Delta_{1}=\Delta$ and $\Delta_{2}=0$ in (7), we have

$$
\sup _{\|\Delta\| \leq L}\left\|M_{T}(\Delta)-M_{T}(0)-\left\{E\left(M_{T}(\Delta)\right)-E\left(M_{T}(0)\right)\right\}\right\|=o_{p}(1) .
$$

Using Assumptions 2(i) and 2(iii), it can be shown that $E\left(M_{T}(\Delta)\right)-E\left(M_{T}(0)\right) \rightarrow-q^{-1} Q_{0} \Delta$ where $Q_{0}=E\left\{f\left(0 \mid x_{t}\right) x_{t} x_{t}^{\prime}\right\}$. A detailed proof of this result is provided in Kim and Muller (2003). 
Therefore, by replacing $E\left(M_{T}(\Delta)\right)-E\left(M_{T}(0)\right)$ with its limit $-q^{-1} Q_{0} \Delta$, we obtain the following result:

$$
\sup _{\|\Delta\| \leq L}\left\|M_{T}(\Delta)-M_{T}(0)+q^{-1} Q_{0} \Delta\right\|=o_{p}(1) .
$$

Hence, (9) is now verified. We now show how it can be used to introduce the first stage estimators.

Let $\hat{\Delta}_{0}=(q-1) \sqrt{T}\left(\hat{\pi}-\pi_{0}\right)+\sqrt{T}\left(\hat{\Pi}-\Pi_{0}\right) \gamma_{0}$. Then, $\hat{\Delta}_{0}=O_{p}(1)$ since $\sqrt{T}\left(\hat{\pi}-\pi_{0}\right)=O_{p}(1)$ and $\sqrt{T}\left(\hat{\Pi}-\Pi_{0}\right)=O_{p}(1)$, given Assumptions 1 and 2. Hence, from the result in (9), we have $M_{T}\left(\hat{\Delta}_{0}\right)=M_{T}(0)-q^{-1} Q_{0} \hat{\Delta}_{0}+o_{p}(1)$, which in turn implies that

$$
M_{T}\left(\hat{\Delta}_{0}\right)=O_{p}(1)
$$

since $M_{T}(0)=O_{p}(1)$ and $q^{-1} Q_{0} \hat{\Delta}_{0}=O_{p}(1)$. Now let

$$
\hat{\Delta}_{1}(\delta)=H(\hat{\Pi}) \delta+\hat{\Delta}_{0}
$$

where $\delta \in R^{G+K_{1}}$. For some finite $L_{1}>0$, then it is straightforward to show that (9) implies that

$$
\sup _{\|\delta\| \leq L_{1}}\left\|M_{T}\left(\hat{\Delta}_{1}(\delta)\right)-M_{T}(0)+q^{-1} Q_{0} \hat{\Delta}_{1}(\delta)\right\|=o_{p}(1) .
$$

Define $\tilde{M}_{T}(\delta)=H(\hat{\Pi})^{\prime} M_{T}\left(\hat{\Delta}_{1}(\delta)\right)$ and $\|H(\hat{\Pi})\|^{2}=\operatorname{tr}\left(H(\hat{\Pi}) H(\hat{\Pi})^{\prime}\right)=O_{p}(1)$. Using the fact that $\hat{\Pi}-\Pi_{0}=o_{p}(1)$ and using the majoring $\left\|\tilde{M}_{T}(\delta)-H\left(\Pi_{0}\right)^{\prime} M_{T}\left(\hat{\Delta}_{0}\right)+q^{-1} Q_{z z} \delta\right\| \leq\|H(\hat{\Pi})\| . \| M_{T}\left(\hat{\Delta}_{1}\right)-$ $M_{T}(0)+q^{-1} Q_{z z} \hat{\Delta}_{1}\|+\| H(\hat{\Pi})-H\left(\Pi_{0}\right)\|.\| M_{T}(0) \|$

$+\left\|H(\hat{\Pi})-H\left(\Pi_{0}\right)\right\| \cdot\left\{\|H(\hat{\Pi})\|+\left\|H\left(\Pi_{0}\right)\right\|\right\} \cdot\left\|q^{-1} Q_{0}\right\| .\|\delta\|$, similarly to Powell (1983), it is shown that (10) and (12) together imply that

$$
\sup _{\|\delta\| \leq L_{1}}\left\|\tilde{M}_{T}(\delta)-H\left(\Pi_{0}\right)^{\prime} M_{T}\left(\hat{\Delta}_{0}\right)+q^{-1} Q_{z z} \delta\right\|=o_{p}(1)
$$

where $Q_{z z}=H\left(\Pi_{0}\right)^{\prime} Q_{0} H\left(\Pi_{0}\right)$. We now want to exploit this result with $\hat{\delta}=T^{1 / 2}(\hat{\alpha}-\alpha)$ instead of $\delta$. For this, we need to show that $\hat{\delta}=O_{P}(1)$. This is done by using Lemma A.4. in Koenker and Zhao (1996), which can be applied under the following conditions ${ }^{7}$ :

(i) $-\delta^{\prime} \tilde{M}_{T}(\lambda \delta) \geq-\delta^{\prime} \tilde{M}_{T}(\delta)$ for $\lambda \geq 1$.

(ii) $\left\|H\left(\Pi_{0}\right)^{\prime} M_{T}\left(\hat{\Delta}_{0}\right)\right\|=O_{p}(1)$.

(iii) $\tilde{M}_{T}(\hat{\delta})=o_{p}(1)$, where $\hat{\delta}=T^{1 / 2}\left(\hat{\alpha}-\alpha_{0}\right)$.

When these conditions are met, $\hat{\delta}=O_{p}(1)$ and therefore $\hat{\delta}=q Q_{z z}^{-1} H\left(\Pi_{0}\right)^{\prime} M_{T}\left(\hat{\Delta}_{0}\right)+o_{p}(1)$. Condition (i) is satisfied since $\psi_{\theta}(u)$ is a function increasing in $u$ and condition (ii) is met by (10) and because $\hat{\Pi}$ is consistent. To show the validality of (iii), we note that

$$
\tilde{M}_{T}(\hat{\delta})=\left[\left.\frac{\partial S_{T}}{\partial \alpha}\right|_{\alpha=\hat{\alpha}}\right]_{-}
$$

where $\left[\left.\frac{\partial S_{T}}{\partial \alpha}\right|_{\alpha=\hat{\alpha}}\right]_{-}$is $o_{p}(1)$ because it is the vector of left-hand-side partial derivatives of the objective function $S_{T}$ evaluated at the solution $\hat{\alpha}$. Hence, $\tilde{M}_{T}(\hat{\delta})=o_{p}(1)$ and all three conditions are now verified. Therefore, we have

$$
T^{1 / 2}\left(\hat{\alpha}-\alpha_{0}\right)=q Q_{z z}^{-1} H\left(\Pi_{0}\right)^{\prime} M_{T}\left(\hat{\Delta}_{0}\right)+o_{p}(1)
$$

\footnotetext{
${ }^{7}$ It is straightforward to adapt the proof in Koenker and Zhao (1996) by replacing $f\left(F^{-1}(\tau)\right) \lambda_{1}(D)$, where $\lambda_{1}(D)$ is the largest eigen-value of $D$, with $\lambda_{1}\left(Q_{z z}\right)$.
} 
which delivers the the preliminary asymptotic representation ${ }^{8}$ for the $\operatorname{DSQR}(\theta, q)$ :

$$
\begin{aligned}
T^{1 / 2}\left(\hat{\alpha}-\alpha_{0}\right)= & Q_{z z}^{-1} H\left(\Pi_{0}\right)^{\prime}\left\{T^{-1 / 2} \sum_{t=1}^{T} q x_{t} \psi_{\theta}\left(v_{t}\right)\right. \\
& \left.+(1-q) Q_{0} T^{1 / 2}\left(\hat{\pi}-\pi_{0}\right)-Q_{0} T^{1 / 2}\left(\hat{\Pi}-\Pi_{0}\right) \gamma_{0}\right\}+o_{p}(1) .
\end{aligned}
$$

Substituting the asymptotic representations $T^{1 / 2}\left(\hat{\pi}-\pi_{0}\right)=Q_{0}^{-1} T^{-1 / 2} \sum_{t=1}^{T} x_{t} \psi_{\theta}\left(v_{t}\right)+o_{p}(1)$ and $T^{1 / 2}\left(\hat{\Pi}_{j}-\Pi_{0 j}\right)=Q_{j}^{-1} T^{-1 / 2}$

$\sum_{t=1}^{T} x_{t} \psi_{\theta}\left(V_{j t}\right)+o_{p}(1)$ into (14) shows that the first term involving $q$ cancels out with the asymptotic representation of $T^{1 / 2}\left(\hat{\pi}-\pi_{0}\right)$ multiplied by $-q Q_{0}$. Simplifying gives

$$
\begin{aligned}
T^{1 / 2}\left(\hat{\alpha}-\alpha_{0}\right)= & T^{-1 / 2} \sum_{t=1}^{T} Q_{z z}^{-1} H\left(\Pi_{0}\right)^{\prime} x_{t} \psi_{\theta}\left(v_{t}\right) \\
& -T^{-1 / 2} \sum_{i=1}^{G} \sum_{t=1}^{T} Q_{z z}^{-1} H\left(\Pi_{0}\right)^{\prime} Q_{0} Q_{i}^{-1} \gamma_{0 i} x_{t} \psi_{\theta}\left(V_{i t}\right)+o_{p}(1) \\
= & D T^{-1 / 2} \sum_{t=1}^{T} Z_{t}+o_{p}(1)
\end{aligned}
$$

where $Z_{t}=\psi_{\theta}\left(W_{t}\right) \otimes x_{t}, \psi_{\theta}\left(W_{t}\right)=\left(\psi_{\theta}\left(v_{t}\right), \psi_{\theta}\left(V_{1 t}\right), \ldots, \psi_{\theta}\left(V_{G t}\right)\right)^{\prime}$ and

$D=Q_{z z}^{-1} H\left(\Pi_{0}\right)^{\prime}\left[I,-Q_{0} Q_{1}^{-1} \gamma_{01}, \ldots,-Q_{0} Q_{G}^{-1} \gamma_{0 G}\right]$. The expression in (15) is a scaled sample mean to which we can apply a CLT provided that $E\left(Z_{t}\right)=0$, which is satisfied by Assumption 2(iv). Here, $E\left(Z_{t}\right)=0$ is satisfied because the same quantile is used in both stages. Now, we examine what happens when LS estimation or a different quantile are used in the first stage. We first consider LS estimation. The corresponding asymptotic representations are $T^{1 / 2}\left(\hat{\pi}^{L S}-\pi_{0}\right)=$ $Q^{-1} T^{-1 / 2} \sum_{t=1}^{T} x_{t} v_{t}+o_{p}(1)$ and $T^{1 / 2}\left(\hat{\Pi}^{L S}-\Pi_{0}\right) \gamma_{0}=Q^{-1} T^{-1 / 2} \sum_{t=1}^{T} x_{t} V_{t} \gamma_{0}+o_{p}(1)$, where $Q=$ $E\left(x_{t} x_{t}^{\prime}\right)$. Substitution of these expressions into (14) gives $T^{1 / 2}\left(\hat{\alpha}-\alpha_{0}\right)=D^{L S} T^{-1 / 2} \sum_{t=1}^{T} Z_{t}^{L S}+$ $o_{p}(1)$, where $D^{L S}=Q_{z z}^{-1} H\left(\Pi_{0}\right)^{\prime}\left[q I,(1-q) Q_{0} Q^{-1},-Q_{0} Q^{-1}\right]$ and $Z_{t}^{L S}=\left(x_{t}^{\prime} \psi_{\theta}\left(v_{t}\right), x_{t}^{\prime} v_{t}\right.$, $\left.x_{t}^{\prime} V_{t} \gamma_{0}\right)^{\prime}$. But, $E\left(Z_{t}^{L S}\right) \neq 0$ because $E\left\{\psi_{\theta}\left(v_{t}\right) \mid x_{t}\right\}=0$ and $E\left\{v_{t} \mid x_{t}\right\}=0$ do not generally hold simultaneously unless $\theta=1 / 2$ and for symmetric distributions. Next, we investigate the consequence of using different quantiles ( $\theta_{1}$ for the first stage and $\theta_{2}$ for the second stage). The asymptotic representations for the first step estimators are given by $T^{1 / 2}\left(\hat{\pi}^{Q}-\pi_{0}\right)=Q_{0}^{-1} T^{-1 / 2} \sum_{t=1}^{T} x_{t} \psi_{\theta_{1}}\left(v_{t}\right)+o_{p}(1)$ and $T^{1 / 2}\left(\hat{\Pi}_{j}^{Q}-\Pi_{0 j}\right)=Q_{j}^{-1} T^{-1 / 2} \sum_{t=1}^{T} x_{t} \psi_{\theta_{1}}\left(V_{j t}\right)+o_{p}(1)$. Plugging these representations into (14) results in $T^{1 / 2}\left(\hat{\alpha}-\alpha_{0}\right)=D^{Q^{2}} T^{-1 / 2} \sum_{t=1}^{T} Z_{t}^{Q}+o_{p}(1)$, where $D^{Q}=Q_{z z}^{-1} H\left(\Pi_{0}\right)^{\prime}[q I,(1-$ q) $\left.I,-Q_{0} Q_{1}^{-1} \gamma_{01}, \ldots,-Q_{0} Q_{G}^{-1} \gamma_{0 G}\right]$ and $Z_{t}^{Q}=\left(x_{t}^{\prime} \psi_{\theta_{2}}\left(v_{t}\right), x_{t}^{\prime} \psi_{\theta_{1}}\left(v_{t}\right), x_{t}^{\prime} \psi_{\theta_{1}}\left(V_{1 t}\right), \ldots, x_{t}^{\prime} \psi_{\theta_{1}}\left(V_{G t}\right)\right)^{\prime}$. Again, $E\left(Z_{t}^{Q}\right) \neq 0$ because $E\left\{\psi_{\theta_{1}}\left(v_{t}\right) \mid x_{t}\right\}=0$ and $E\left\{\psi_{\theta_{2}}\left(v_{t}\right) \mid x_{t}\right\}=0$ cannot hold unless $\theta_{1}=\theta_{2}$. This issue of asymptotic bias caused by $E\left(Z_{t}\right) \neq 0$ is analysed in Kim and Muller (2000).

\section{B. APPENDIX. PROOFS}

Proof of Proposition 1: Recalling Theorem 3.2 in Koenker and Bassett (1978), we have $\delta(\theta, \lambda y, X)=$ $\lambda \delta(\theta, y, X)$ for any $\lambda>0$ and $\delta(\theta, y+X \gamma, X)=\delta(\theta, y, X)+\gamma$, where $\gamma$ is a parameter of appropriate dimension. The above invariance properties imply that $\delta(\theta, q y+(1-q) X H(\hat{\Pi}) \tilde{\alpha}, X H(\hat{\Pi}))$

\footnotetext{
${ }^{8}$ An alternative approach would have been to work with an empirical process for the objective function itself: $\zeta_{T}(\Delta)=\sum_{t=1}^{T}\left[\rho_{\theta}\left(q v_{t}-T^{-1 / 2} x_{t}^{\prime} \Delta\right)-\rho_{\theta}\left(q v_{t}\right)\right]$ and to use the method proposed in Pollard (1991).
} 
$=\delta(\theta, q y, X H(\hat{\Pi}))+(1-q) \tilde{\alpha}=q \delta(\theta, y, X H(\hat{\Pi}))+(1-q) \tilde{\alpha}=q \tilde{\alpha}+(1-q) \tilde{\alpha}=\tilde{\alpha}$. This is result (i). If $K_{2}=G$ and $H(\hat{\Pi})$ is of full column rank, then $H(\hat{\Pi})$ is non singular. Using $\delta(\theta, y, X A)=$ $A^{-1} \delta(\theta, y, X)$ for any non singular matrix $A$ (Theorem 3.2 in Koenker and Bassett, 1978), we obtain $\tilde{\alpha}=\delta(\theta, y, X H(\hat{\Pi}))=H(\hat{\Pi})^{-1} \delta(\theta, y, X)=H(\hat{\Pi})^{-1} \hat{\pi}$. Next we can show that $\tilde{\alpha}=\hat{\alpha}$ because using (i) we have $\tilde{\alpha}=\delta(\theta, q y+(1-q) X H(\hat{\Pi}) \tilde{\alpha}, X H(\hat{\Pi}))=\delta(\theta, q y+(1-q) X \hat{\pi}, X H(\hat{\Pi}))=\hat{\alpha}$. This shows (ii). QED.

Proof of Proposition 2: Consider (15). Since $Z_{t}$ is i.i.d. by Assumption 1, it is sufficient to show that $\operatorname{var}\left(Z_{t}\right)$ is bounded to apply the Lindeberg-Levy's CLT. The moment condition on $x_{t}$ in Assumption 2(i) is sufficient for this purpose because $\psi_{\theta}(\cdot)^{2}$ is bounded by 1 . Noting that $\operatorname{var}\left(Z_{t}\right)=\Omega$, we have $T^{-1 / 2} \sum_{t=1}^{T} Z_{t} \stackrel{d}{\rightarrow} N(0, \Omega)$, which proves the claim in the proposition. QED. Proof of Proposition 3: We first prove the claim $\hat{\Omega} \stackrel{p}{\rightarrow} \Omega$. Consider the (1,1)-submatrices of $\hat{\Omega}$ and $\Omega$, which are given by $\hat{\Omega}_{11}=T^{-1} \sum_{t=1}^{T} \psi_{\theta}\left(\hat{v}_{t}\right)^{2} x_{t} x_{t}^{\prime}$ and $\Omega_{11}=E\left\{\psi_{\theta}\left(v_{t}\right)^{2} x_{t} x_{t}^{\prime}\right\}$. The consistency of $\hat{\Omega}_{11}$ is proved in two steps: (i) $\left|\Omega_{11 T}-\Omega_{11}\right|=o_{p}(1)$ and (ii) $\left|\hat{\Omega}_{11}-\Omega_{11 T}\right|=o_{p}(1)$ where $\Omega_{11 T}=T^{-1} \sum_{t=1}^{T} \psi_{\theta}\left(v_{t}\right)^{2} x_{t} x_{t}^{\prime}$. The first step is obtained by applying the LLN for i.i.d. random variables under Assumptions 1 and 2(i). We now prove the second step. Consider the $(i, j)$ component of $\left|\hat{\Omega}_{11}-\Omega_{11 T}\right|$ which is given by $\left|T^{-1} \sum_{t=1}^{T}\left\{\psi_{\theta}\left(\hat{v}_{t}\right)-\psi_{\theta}\left(v_{t}\right)\right\}\left\{\psi_{\theta}\left(\hat{v}_{t}\right)+\psi_{\theta}\left(v_{t}\right)\right\} x_{t i} x_{t j}\right|$ $\leq 2 T^{-1} \sum_{t=1}^{T}\left|\psi_{\theta}\left(\hat{v}_{t}\right)-\psi_{\theta}\left(v_{t}\right)\right|\left|x_{t i}\right|\left|x_{t j}\right| \leq 2 T^{-1} \sum_{t=1}^{T} 1_{\left[\left|v_{t}\right| \leq d_{T}\right]}\left|x_{t i}\right|\left|x_{t j}\right|$, where $d_{T}=\left\|x_{t}\right\| \times\left\|\hat{\pi}-\pi_{0}\right\|$. The first inequality comes from Minkowski's inequality and $\left|\psi_{\theta}(\cdot)\right| \leq 1$ and the second inequality is obtained using $v_{t}-\hat{v}_{t}=x_{t}^{\prime}\left(\hat{\pi}-\pi_{0}\right),\left|x_{t}^{\prime}\left(\hat{\pi}-\pi_{0}\right)\right| \leq|| x_{t}|| \times|| \hat{\pi}-\pi_{0}||$ and the fact that $\left|1_{[x \leq 0]}-1_{[y \leq 0]}\right| \leq$ $1_{[|x| \leq|x-y|]}$. Let $U_{T}=T^{-1} \sum_{t=1}^{T} 1_{\left[\left|v_{t}\right| \leq d_{T}\right]}\left|x_{t i}\right|\left|x_{t j}\right|$ and consider a set $A=\left\{U_{T}>\eta\right\}$ for $\eta>0$. For any event $B$, we have $P(A) \leq P(A \cap B)+P\left(B^{c}\right)$. We choose $B=\left\{\left\|\hat{\pi}-\pi_{0}\right\| \leq z T^{-d}\right\}$ where $z>0$ and $0<d<1 / 2$. Then, we have $P\left(B^{c}\right) \rightarrow 0$ since $T^{1 / 2}\left(\hat{\pi}-\pi_{0}\right)=O_{p}(1)$. Now consider

$$
\begin{aligned}
P(A \cap B) \leq & (\eta T)^{-1} \sum_{t=1}^{T} E\left\{\int_{-\| x_{t}|| z T^{-d}}^{\| x_{t}|| z T^{-d}} f\left(\lambda \mid x_{t}\right) d \lambda\left|x_{t i}\right|\left|x_{t j}\right|\right\} \\
& \text { (by the generalised Cebyshev inequality) } \\
\leq & (\eta T)^{-1} \sum_{t=1}^{T} E\left\{\int_{-|| x_{t} \| z T^{-d}}^{\| x_{t}|| z T^{-d}} f_{0} d \lambda\left|x_{t i} \| x_{t j}\right|\right\} \\
& (\text { by Assumption } 3(\mathrm{i})) \\
= & 2 z f_{0} \eta^{-1} T^{-d} E\left\{\left\|x_{t}\right\|\left|x_{t i} \| x_{t j}\right|\right\}
\end{aligned}
$$

The last expression converges to zero because $E\left\{|| x_{t}||\left|x_{t i}\right|\left|x_{t j}\right|\right\}<\infty$ by Assumption 2(i). Hence, we have proved that $U_{T}=T^{-1} \sum_{t=1}^{T} 1_{\left[\left|v_{t}\right| \leq d_{T}\right]}\left|x_{t i}\right|\left|x_{t j}\right| \stackrel{p}{\rightarrow} 0$ which in turn implies that $\left|\hat{\Omega}_{11}-\Omega_{11 T}\right|=$ $o_{p}(1)$. The second step is now proved. By combining (i) and (ii), we have $\left|\hat{\Omega}_{11}-\Omega_{11}\right|=o_{p}(1)$. The same argument can be applied to all other diagonal and off-diagonal terms of $\hat{\Omega}$ to show their consistency. Therefore, $|\hat{\Omega}-\Omega|=o_{p}(1)$.

Next, we turn to the claim $|\hat{D}-D|=o_{p}(1)$. We need to show the consistency of $\hat{Q}_{0}, \hat{Q}_{1}, \ldots, \hat{Q}_{G}, \hat{\Pi}$ and $\hat{\gamma}$. Since the results $\hat{\Pi}-\Pi_{0}=o_{p}(1)$ and $\hat{\gamma}-\gamma_{0}=o_{p}(1)$ are trivial, we first focus on $\left|\hat{Q}_{0}-Q_{0}\right|=o_{p}(1)$. Let be $Q_{0 T}=\left(2 c_{0 T} T\right)^{-1} \sum_{t=1}^{T} 1_{\left[-c_{0 T} \leq v_{t} \leq c_{0 T}\right]} x_{t} x_{t}^{\prime}$ and $\tilde{Q}_{0 T}=\left(2 c_{0 T} T\right)^{-1}$ $\sum_{t=1}^{T} 1_{\left[-\hat{c}_{0 T} \leq \hat{v}_{t} \leq \hat{c}_{0 T}\right]} x_{t} x_{t}^{\prime}$. As before, the proof is carried out in three steps: (i) $\left|Q_{0 T}-Q_{0}\right|=o_{p}(1)$, (ii) $\left|\tilde{Q}_{0 T}-Q_{0 T}\right|=o_{p}(1)$ and (iii) $\left|\tilde{Q}_{0 T}-\hat{Q}_{0 T}\right|=o_{p}(1)$.

We start with (i). The Mean value theorem implies that $E\left(Q_{0 T}\right)=E\left\{T^{-1} \sum_{t=1}^{T} f\left(\xi_{T} \mid x_{t}\right) x_{t} x_{t}^{\prime}\right\}$, where $-c_{0 T} \leq \xi_{T} \leq c_{0 T}$. Noting that $\xi_{T}=o_{p}(1),\left|E\left(Q_{0 T}\right)-Q_{0}\right|=o(1)$ by Minkowski inequality and Assumptions 2(i) and 2(ii). Using a LLN for double arrays, we have $\left|Q_{0 T}-E\left(Q_{0 T}\right)\right|=o_{p}(1)$. Therefore, the first step is proven. 
Now we turn to (ii). Using the fact that $\left|1_{[x \leq 0]}-1_{[y \leq 0]}\right| \leq 1_{[|x| \leq|x-y|]}$, the $(i, j)^{t h}$ element of $\left|\tilde{Q}_{0 T}-Q_{0 T}\right|$ given by $\left|\left(2 c_{0 T} T\right)^{-1} \sum_{t=1}^{T}\left(1_{\left[-\hat{c}_{0 T} \leq \hat{v}_{t} \leq \hat{c}_{0 T}\right]}-1_{\left[-c_{0 T} \leq v_{t} \leq c_{0 T}\right]}\right) x_{t} x_{t}^{\prime}\right|$ is bounded by $\left(2 c_{0 T} T\right)^{-1}$ $\sum_{t=1}^{T}\left(1_{\left[\left|v_{t}+c_{0 T}\right| \leq d_{T}\right]}+1_{\left[\left|v_{t}-c_{0 T}\right| \leq d_{T}\right]}\right)$

$\left|x_{t i}\right|\left|x_{t j}\right|=U_{1 T}+U_{2 T}$, where $d_{T}=\| x_{t}|| \times|| \hat{\pi}-\pi_{0}||+\left|\hat{c}_{0 T}-c_{0 T}\right|, U_{1 T}=\left(2 c_{0 T} T\right)^{-1} \sum_{t=1}^{T} 1_{\left[\left|v_{t}+c_{0 T}\right| \leq d_{T}\right]}\left|x_{t i}\right|\left|x_{t j}\right|$ and $U_{2 T}=\left(2 c_{0 T} T\right)^{-1} \sum_{t=1}^{T} 1_{\left[\left|v_{t}-c_{0 T}\right| \leq d_{T}\right]}\left|x_{t i}\right|\left|x_{t j}\right|$. By using the same argument used to show $U_{T} \rightarrow 0$ in the proof of $\left|\hat{\Omega}_{11}-\Omega_{11 T}\right|=o_{p}(1)$, one can show $U_{1 T}=o_{p}(1)$ and $U_{2 T}=o_{p}(1)$, which implies $\left|\tilde{Q}_{0}-Q_{0}\right|=o_{p}(1)$. The second step is proven.

To show step (iii), we note that $\hat{Q}_{0 T}-\tilde{Q}_{0 T}=\left(c_{0 T} / \hat{c}_{0 T}-1\right) \tilde{Q}_{0 T}$. Since $\tilde{Q}_{0 T}=O_{p}(1)$ and $\left(c_{0 T} / \hat{c}_{0 T}-1\right)=o_{p}(1)$ by Assumption 3(ii), the last step is proved. Therefore, we have: $\left|\hat{Q}_{0}-Q_{0}\right|=$ $o_{p}(1)$. The same argument can be used to show $\left|\hat{Q}_{j}-Q_{j}\right|=o_{p}(1)$ for $j=1, \ldots, G$. Therefore, we have $\hat{D} \stackrel{p}{\rightarrow} D$. QED.

\section{APPENDIX. SIMULATION DESIGN}

The structural system is given by $B\left[\begin{array}{c}y_{t}^{\prime} \\ Y_{t}^{\prime}\end{array}\right]+\Gamma x_{t}^{\prime}=U_{t}^{\prime}$, where $\left[\begin{array}{c}y_{t}^{\prime} \\ Y_{t}^{\prime}\end{array}\right]$ is a $2 \times 1$ vector of endogenous variables, $x_{t}^{\prime}$ is a $4 \times 1$ vector of exogenous variables with the first element set to one, $U_{t}^{\prime}$ is a $2 \times 1$ vector of errors, $B=\left[\begin{array}{cc}1 & -0.5 \\ -0.7 & 1\end{array}\right]$ and $\Gamma=\left[\begin{array}{llll}-1 & -0.2 & 0 & 0 \\ -1 & 0 & -0.4 & -0.5\end{array}\right]$. We are interested in the first equation of the system and the system is over-identified by the zero restrictions $\Gamma_{13}=\Gamma_{14}=\Gamma_{22}=0$. Here, the parameters in (1) are $\gamma_{0}=0.5$ and $\beta_{0}^{\prime}=(1,0.2)$, $X_{1}$ is the first two columns in $X$ and $u$ is the first column in $U$. The above structural equation can be written as $\left[\begin{array}{ll}y & Y\end{array}\right] B^{\prime}=-X \Gamma^{\prime}+U$, which gives the following reduced form equations $\left[\begin{array}{ll}y & Y\end{array}\right]=X\left[\begin{array}{ll}\pi_{0} & \Pi_{0}\end{array}\right]+\left[\begin{array}{ll}v & V\end{array}\right]$, where $\left[\begin{array}{ll}\pi_{0} & \Pi_{0}\end{array}\right]=-\Gamma^{\prime}\left(B^{\prime}\right)^{-1}$ and $\left[\begin{array}{ll}v & V\end{array}\right]=U\left(B^{\prime}\right)^{-1}$. We obtain $\pi_{0}^{\prime}=(2.3,0.3,0.3,-0.15)$ and $\Pi_{0}^{\prime}=(2.6,0.2,0.6,-0.3)$.

The errors $\left[\begin{array}{ll}v & V\end{array}\right]$ in the reduced form equations are generated so that Assumption 2(iv) is satisfied: $v=v^{e}-F_{v^{e}}^{-1}(\theta)$ and $V=V^{e}-F_{V e}^{-1}(\theta)$, where $v^{e}$ and $V^{e}$ are generated for the different simulation sets by using the three distributions $\mathrm{N}(0,1), t(3)$ and $\operatorname{LN}(0,1)$ with correlation -0.1 , and $F_{v^{e}}^{-1}(\theta)$ and $F_{V^{e}}^{-1}(\theta)$ are the inverse cumulative functions of $v^{e}$ and $V^{e}$ evaluated at $\theta$. The second to fourth columns in $X$ are generated using the normal distribution with zero means and covariances, and unit variances. Finally, we generate the endogenous variables $\left[\begin{array}{ll}y & Y\end{array}\right]$ using the reduced-form equations. 
Table A1. Simulation Means and Standard Deviations of $D S Q R(\theta, q=0.1): t(3)$.

\begin{tabular}{|c|c|c|c|c|c|c|c|}
\hline & & $\theta$ & 0.05 & 0.25 & 0.50 & 0.75 & 0.95 \\
\hline \multirow{6}{*}{$T=50$} & \multirow{2}{*}{$\tilde{\gamma}$} & & & & & & \\
\hline & & $\begin{array}{r}\text { Mean } \\
\text { Std }\end{array}$ & $\begin{array}{r}-0.31 \\
1.36\end{array}$ & $\begin{array}{r}-0.03 \\
0.68\end{array}$ & $\begin{array}{l}0.01 \\
0.50\end{array}$ & $\begin{array}{r}-0.04 \\
0.80\end{array}$ & $\begin{array}{r}-0.34 \\
1.34\end{array}$ \\
\hline & \multirow[t]{2}{*}{$\widetilde{\beta}_{0}$} & Mean & 0.15 & 0.00 & -0.01 & 0.24 & 1.93 \\
\hline & & Std & 4.73 & 2.13 & 1.61 & 2.50 & 4.41 \\
\hline & \multirow[t]{2}{*}{$\widetilde{\beta}_{1}$} & Mean & 0.15 & 0.01 & -0.01 & 0.02 & 0.19 \\
\hline & & Std & 1.16 & 0.37 & 0.32 & 0.44 & 1.13 \\
\hline \multirow{6}{*}{$T=300$} & \multirow[t]{2}{*}{$\tilde{\gamma}$} & Mean & -0.07 & 0.00 & -0.01 & 0.00 & -0.06 \\
\hline & & Std & 0.72 & 0.18 & 0.15 & 0.19 & 0.61 \\
\hline & \multirow[t]{2}{*}{$\widetilde{\beta}_{0}$} & Mean & -0.06 & -0.02 & 0.04 & 0.04 & 0.44 \\
\hline & & Std & 2.32 & 0.60 & 0.49 & 0.62 & 2.12 \\
\hline & \multirow[t]{2}{*}{$\widetilde{\beta}_{1}$} & Mean & 0.03 & -0.01 & 0.00 & -0.01 & 0.02 \\
\hline & & Std & 0.39 & 0.13 & 0.11 & 0.14 & 0.39 \\
\hline
\end{tabular}

Table A2. Simulation Means and Standard Deviations of $D S Q R(\theta, q=0.5): t(3)$.

\begin{tabular}{|c|c|c|c|c|c|c|c|}
\hline & & $\theta$ & 0.05 & 0.25 & 0.50 & 0.75 & 0.95 \\
\hline \multirow{6}{*}{$T=50$} & \multirow[t]{2}{*}{$\tilde{\gamma}$} & Mean & -0.33 & -0.02 & 0.00 & -0.04 & -0.35 \\
\hline & & Std & 1.33 & 0.71 & 0.49 & 0.79 & 1.30 \\
\hline & \multirow[t]{2}{*}{$\widetilde{\beta}_{0}$} & Mean & 0.67 & 0.04 & 0.00 & 0.18 & 1.50 \\
\hline & & Std & 4.47 & 2.21 & 1.55 & 2.48 & 4.25 \\
\hline & \multirow{2}{*}{$\widetilde{\beta}_{1}$} & Mean & 0.15 & 0.00 & 0.00 & 0.03 & 0.15 \\
\hline & & Std & 1.12 & 0.36 & 0.31 & 0.43 & 1.09 \\
\hline \multirow{6}{*}{$T=300$} & \multirow[t]{2}{*}{$\tilde{\gamma}$} & Mean & -0.06 & 0.00 & -0.01 & 0.00 & -0.06 \\
\hline & & Std & 0.69 & 0.18 & 0.15 & 0.19 & 0.60 \\
\hline & \multirow[t]{2}{*}{$\widetilde{\beta}_{0}$} & Mean & 0.12 & 0.00 & 0.04 & 0.02 & 0.27 \\
\hline & & Std & 2.21 & 0.60 & 0.49 & 0.61 & 2.07 \\
\hline & \multirow[t]{2}{*}{$\widetilde{\beta}_{1}$} & Mean & 0.03 & -0.01 & 0.00 & -0.01 & 0.02 \\
\hline & & Std & 0.39 & 0.13 & 0.11 & 0.14 & 0.39 \\
\hline
\end{tabular}

Table A3. Simulation Means and Standard Deviations of $D S Q R(\theta, q=0.1): \mathbf{L N}(\mathbf{0 , 1})$.

\begin{tabular}{|c|c|c|c|c|c|c|c|}
\hline & & $\theta$ & 0.05 & 0.25 & 0.50 & 0.75 & 0.95 \\
\hline \multirow{6}{*}{$T=50$} & \multirow[t]{2}{*}{$\widetilde{\gamma}$} & Mean & 0.00 & 0.00 & 0.02 & 0.13 & $0.49(0.46)$ \\
\hline & & Std & 0.07 & 0.10 & 0.25 & 0.69 & $0.35(0.26)$ \\
\hline & \multirow[t]{2}{*}{$\widetilde{\beta}_{0}$} & Mean & -0.08 & -0.05 & 0.00 & 0.08 & $0.48(0.54)$ \\
\hline & & Std & 0.05 & 0.06 & 0.14 & 0.29 & $0.60(0.49)$ \\
\hline & \multirow[t]{2}{*}{$\widetilde{\beta}_{1}$} & Mean & 0.00 & 0.00 & -0.01 & -0.05 & $-0.20(-0.20)$ \\
\hline & & Std & 0.05 & 0.07 & 0.13 & 0.24 & $0.37(0.33)$ \\
\hline \multirow{6}{*}{$T=300$} & \multirow[t]{2}{*}{$\tilde{\gamma}$} & Mean & 0.00 & 0.00 & 0.00 & 0.00 & $0.29(0.30)$ \\
\hline & & Std & 0.02 & 0.04 & 0.06 & 0.14 & $0.45(0.25)$ \\
\hline & \multirow[t]{2}{*}{$\widetilde{\beta}_{0}$} & Mean & -0.08 & -0.06 & -0.03 & 0.04 & $0.40(0.43)$ \\
\hline & & Std & 0.01 & 0.02 & 0.05 & 0.10 & $0.42(0.42)$ \\
\hline & \multirow[t]{2}{*}{$\widetilde{\beta}_{1}$} & Mean & 0.00 & 0.00 & 0.00 & 0.00 & $-0.11(-0.13)$ \\
\hline & & Std & 0.02 & 0.03 & 0.05 & 0.10 & $0.32(0.25)$ \\
\hline
\end{tabular}


Table A4. Simulation Means and Standard Deviations of $D S Q R(\theta, q=0.5): \mathbf{L N}(\mathbf{0 , 1})$.

\begin{tabular}{|c|c|c|c|c|c|c|c|}
\hline & & $\theta$ & 0.05 & 0.25 & 0.50 & 0.75 & 0.95 \\
\hline \multirow{6}{*}{$T=50$} & \multirow{2}{*}{$\tilde{\gamma}$} & & & & & 0.1 & \\
\hline & & $\begin{array}{r}\text { Mean } \\
\text { Std }\end{array}$ & 0.00 & $\begin{array}{r}0.00 \\
0.10\end{array}$ & 0.02 & 0.11 & $\begin{array}{l}0.48(0.4 /) \\
0.43(0.29)\end{array}$ \\
\hline & \multirow{2}{*}{$\widetilde{\beta}_{0}$} & Mean & -0.06 & -0.05 & -0.02 & 0.02 & $0.36(0.41)$ \\
\hline & & Std & 0.04 & 0.06 & 0.15 & 0.26 & $0.75(0.58)$ \\
\hline & \multirow[t]{2}{*}{$\widetilde{\beta}_{1}$} & Mean & 0.00 & 0.00 & -0.01 & -0.04 & $-0.19(-0.20)$ \\
\hline & & Std & 0.05 & 0.07 & 0.13 & 0.25 & $0.45(0.45)$ \\
\hline \multirow{6}{*}{$T=300$} & \multirow[t]{2}{*}{$\tilde{\gamma}$} & Mean & 0.00 & 0.00 & 0.00 & 0.00 & $0.29(0.29)$ \\
\hline & & Std & 0.02 & 0.04 & 0.06 & 0.15 & $0.49(0.29)$ \\
\hline & \multirow[t]{2}{*}{$\widetilde{\beta}_{0}$} & Mean & -0.08 & -0.06 & -0.03 & 0.02 & $0.29(0.32)$ \\
\hline & & Std & 0.01 & 0.02 & 0.05 & 0.10 & $0.44(0.40)$ \\
\hline & \multirow[t]{2}{*}{$\widetilde{\beta}_{1}$} & Mean & 0.00 & 0.00 & 0.00 & 0.00 & $-0.10(-0.12)$ \\
\hline & & Std & 0.02 & 0.03 & 0.05 & 0.11 & $0.37(0.31)$ \\
\hline
\end{tabular}

Table A5. Simulation Means and Standard Deviations of $D S L S$ with a single outlier: $\mathbf{N}(\mathbf{0 , 1})$.

\begin{tabular}{|c|c|c|c|c|c|}
\hline & & $\begin{array}{l}\text { Standard } \\
\text { Measures }\end{array}$ & & $\begin{array}{l}\text { Robust } \\
\text { Measures }\end{array}$ & \\
\hline & & & & & \\
\hline \multirow{6}{*}{$T=50$} & \multirow[t]{2}{*}{$\tilde{\gamma}$} & Mean & 0.24 & Median & 0.03 \\
\hline & & Std & 2.08 & IQR & 2.18 \\
\hline & \multirow[t]{2}{*}{$\widetilde{\beta}_{0}$} & Mean & 0.23 & Median & 0.62 \\
\hline & & Std & 6.51 & IQR & 6.82 \\
\hline & \multirow{2}{*}{$\widetilde{\beta}_{1}$} & Mean & -0.01 & Median & -0.04 \\
\hline & & Std & 1.54 & IQR & 1.65 \\
\hline & & & & & \\
\hline \multirow{6}{*}{$T=300$} & \multirow[t]{2}{*}{$\widetilde{\gamma}$} & Mean & 0.04 & Median & 0.02 \\
\hline & & Std & 0.32 & IQR & 0.40 \\
\hline & \multirow[t]{2}{*}{$\widetilde{\beta}_{0}$} & Mean & 0.04 & Median & 0.10 \\
\hline & & Std & 1.03 & IQR & 1.24 \\
\hline & \multirow{2}{*}{$\widetilde{\beta}_{1}$} & Mean & 0.01 & Median & 0.00 \\
\hline & & Std & 0.25 & IQR & 0.29 \\
\hline
\end{tabular}

Table A6. Simulation Means and Standard Deviations of $D S Q R(\theta, q=1)$ with a single outlier: $\mathbf{N}(\mathbf{0 , 1})$.

\begin{tabular}{|c|c|c|c|c|c|c|c|}
\hline & & $\theta$ & 0.05 & 0.25 & 0.50 & 0.75 & 0.95 \\
\hline \multirow{6}{*}{$T=50$} & \multirow[t]{2}{*}{$\bar{\gamma}$} & Mean & -0.04 & 0.02 & 0.01 & 0.01 & 0.02 \\
\hline & & Std & 0.86 & 0.64 & 0.42 & 0.52 & 1.56 \\
\hline & \multirow[t]{2}{*}{$\widetilde{\beta}_{0}$} & Mean & 0.12 & -0.05 & 0.00 & 0.02 & 0.53 \\
\hline & & Std & 2.89 & 2.05 & 1.33 & 1.67 & 4.91 \\
\hline & \multirow[t]{2}{*}{$\widetilde{\beta}_{1}$} & Mean & 0.03 & 0.00 & 0.00 & 0.00 & 0.01 \\
\hline & & Std & 0.53 & 0.31 & 0.27 & 0.33 & 1.47 \\
\hline \multirow{6}{*}{$T=300$} & \multirow{2}{*}{$\tilde{\gamma}$} & Мегп & 001 & 001 & 001 & 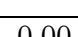 & 000 \\
\hline & & Std & 0.25 & 0.15 & 0.14 & 0.15 & 0.24 \\
\hline & \multirow[t]{2}{*}{$\widetilde{\beta}_{0}$} & Mean & -0.03 & -0.02 & -0.02 & -0.01 & 0.03 \\
\hline & & Std & 0.81 & 0.49 & 0.46 & 0.50 & 0.79 \\
\hline & \multirow[t]{2}{*}{$\widetilde{\beta}_{1}$} & Mean & 0.00 & 0.00 & 0.00 & 0.00 & 0.01 \\
\hline & & Std & 0.18 & 0.11 & 0.10 & 0.11 & 0.17 \\
\hline
\end{tabular}


Table A7. Simulation Means and Standard Deviations of $D S Q R(\theta, q=1)$ with a single outlier: $\mathbf{N}(\mathbf{0 , 1})$.

\begin{tabular}{|c|c|c|c|c|c|c|c|}
\hline & & $\theta$ & 0.05 & 0.25 & 0.50 & 0.75 & 0.95 \\
\hline \multirow{6}{*}{$T=50$} & \multirow{2}{*}{$\tilde{\gamma}$} & & & & & & \\
\hline & & $\begin{array}{c}\text { Median } \\
\text { IQR }\end{array}$ & $\begin{array}{r}-0.13 \\
0.72\end{array}$ & $\begin{array}{r}-0.04 \\
0.48\end{array}$ & $\begin{array}{r}-0.04 \\
0.44\end{array}$ & $\begin{array}{r}-0.05 \\
0.49\end{array}$ & $\begin{array}{r}-0.11 \\
1.04\end{array}$ \\
\hline & \multirow[t]{2}{*}{$\widetilde{\beta}_{0}$} & Median & 0.46 & 0.15 & 0.15 & 0.21 & 0.56 \\
\hline & & $\mathrm{IQR}$ & 2.41 & 1.57 & 1.43 & 1.57 & 3.18 \\
\hline & \multirow[t]{2}{*}{$\widetilde{\beta}_{1}$} & Median & 0.05 & 0.01 & 0.02 & 0.01 & 0.08 \\
\hline & & IQR & 0.57 & 0.35 & 0.32 & 0.36 & 0.86 \\
\hline \multirow{6}{*}{$T=300$} & \multirow[t]{2}{*}{$\tilde{\gamma}$} & Median & -0.02 & -0.01 & 0.00 & -0.01 & -0.01 \\
\hline & & IQR & 0.31 & 0.20 & 0.18 & 0.19 & 0.31 \\
\hline & \multirow[t]{2}{*}{$\widetilde{\beta}_{0}$} & Median & 0.03 & 0.03 & 0.00 & 0.02 & 0.09 \\
\hline & & $\mathrm{IQR}$ & 1.01 & 0.65 & 0.58 & 0.62 & 1.01 \\
\hline & \multirow[t]{2}{*}{$\widetilde{\beta}_{1}$} & Median & 0.01 & 0.00 & 0.00 & 0.01 & 0.01 \\
\hline & & IQR & 0.22 & 0.14 & 0.13 & 0.15 & 0.22 \\
\hline
\end{tabular}

